\title{
GDV as a method of rapid diagnostics and monitoring of the psychophysiological state of schoolchildren
}

\author{
Marina Skuratovskaya ${ }^{1, *}$, Nadezda Manohina ${ }^{1}$, Larisa Kobrina $^{2}$, Mihail Yanovsky ${ }^{3}$, and \\ Sergei Dzura ${ }^{4}$ \\ ${ }^{1}$ Don State Technical University, 344003, Gagarina str., 1, Rostov-on-don, Russia \\ ${ }^{2}$ Pushkin Leningrad State University, 196605, 10, shosse Peterburgskoe, Pushkin, Saint Petersburg, \\ Russia \\ ${ }^{3}$ Donetsk National University, 283001, Universitetskaya str., 24, Donetsk, Ukraine \\ ${ }^{4}$ Donetsk National Technical University, 283001, Artema str., 58, Donetsk, Ukraine
}

\begin{abstract}
The article reveals the essence of the gas-discharge visualization method. It shows the historical path of development of research in this area over the time period of the XIX-XXI centuries. The article presents a range of modern research areas that justify the possibility of using the method of gas-discharge visualization (Bioelectrography) in medicine, sports, agriculture, criminology, psychology and pedagogy. The results of the study are presented in the process of using the method of gasdischarge visualization (Bioelectrography) in speech therapy, as well as in monitoring speech correction and statokinetic functions.
\end{abstract}

\section{Introduction}

Impaired speech development is one of the most common forms of dysontogenesis in a child's development. Speech underdevelopment is one of the reason of communicative difficulties. As a result, it has a negative impact on the emotional, volitional and personal spheres on the child. During the learning, the children often have specific reading and writing disorders - dysgraphia and dyslexia. Mastering oral and written speech difficulties are often caused by impaired motor functions. Static and dynamic coordination of movements, kinetic organization of the motor act are violated [1-4]. Overcoming a child's speech disorders and accompanying motor function disorders often requires considerable effort. It is based on an accurate knowledge of the features of the child's speech and psychophysical development. Psychological and pedagogical diagnostics is the main direction in speech therapy [5-6].

Nowadays, modern software and hardware complexes are increasingly used in psychological and pedagogical research to solve diagnostic problems. They allow you to ensure the efficiency, clarity and reliability of diagnostics, the possibility of dynamic monitoring of the correctional work results to overcome speech disorders and concomitant

\footnotetext{
* Corresponding author: marinasku@yandex.ru
} 
disorders of psychophysical development. One of these innovative methods, which has been actively included in psychological and pedagogical research in recent years, is the method of gas-discharge visualization (GDV) (Bioelectrography). The gas-discharge visualization method is based on the Kirlian effect. This method makes it possible to register, analyze and computer process the features of the glow that occurs near the surface of an object (human fingertips) when it is placed in an electric field with amplification in a gas discharge. The features of the glow depend on the state of the autonomic nervous system, the balance of its sympathetic and parasympathetic divisions, adaptive levels. It allows us to assess the energy state of a person at the psycho-emotional and physiological levels. This information provides additional data for the specialist, necessary at the initial stage to assess the correctness of the choice of correctional work methods, their compliance with the psychophysical characteristics of the child. These data are also useful for monitoring the further dynamics of speech development, as they may indicate the effectiveness of speech therapy correction.

The development of the gas-discharge visualization method has a long history. At the end of the XIX century Nikola Tesla studied the glow phenomenon of biological objects in rarefied gases, using a resonant transformer developed by him. In addition, he studied the physiological effects of high-frequency currents [7]. Nikola Tesla presented the experiment results on the glow of biological objects in high-frequency currents in various European countries. At the same time, the Belarusian scientist Ya. O. Narkevich-Yodko managed to get the first images of biological objects in a gas discharge. He presented the study results at electrography and electrophysiology conferences. At the beginning of the XX century many scientists in Russia and another countries of the world such as Maximilian Tepler and Hans Mal from Germany, Arthur Robert von Hippel from the USA, Sylvester Pratt and Jan Schlemmer from the Czech Republic were engaged in research and registration of the biological objects glowing in photographs. However, the real breakthrough in studying of the biological objects glowing in a gas discharge is associated with the names of Russian scientists S. D. and V. H. Kirlian [7].

In 1949, Russian scientists S. D. and V. H. Kirlian developed and patented the method for photographing objects in high-frequency currents. This method is named after the author of the photo Kirlian. In their work, the scientists noted and demonstrated numerous images. The emission of electrons in a high-frequency electric field was observed. It caused a glow around objects; it is inherent in all biological and non-biological objects. [8].

In the second part of the XX century, Professor V. M. Inyushin from Kazakhstan started systematic research in the field of kirlianography. He put forward the bioplasmic hypothesis to explain the Kirlian effect. Scientific research of this period contributed to the fact that kirlianography was used in medicine, psychology and criminology. In the 60s, A. F. Aubakirov defended his first $\mathrm{PhD}$ thesis on the use of kirlianography in criminology. At the same time, scientists from many countries in Europe and America conducted research and developed devices based on the Kirlian effect. In addition, they had numerous conferences and published books on this problem (Peter Tompkins, Stanley Krippner). We can mention Professor from Brazil Newton Oliveira Milhomens (1937-2007) who improved the device for Kirlian photography and used it for research in psychology. Also Thelma Moss and Kendall Johnson (Thelma Moss, Kendall Johnson) and later David Sheikin and Thelma Moss from the United States, Giuseppe Ambrosini (Giuseppe Ambrosini) from Italy began to study using of Kirlian photography in medicine. In Russia, Bioelectrography was used in industry (S. F. Romaniy), medicine (P. E. Erasov), including psychiatry. [7].

In 1978, the International Union of Medical and Applied Bioelectrography (IUMAB International Union of Medical Applied Bioelectrography) was organized. K. G. Korotkov was elected as a President. 
The modern development of the gas-discharge visualization method is associated with the name of K. G. Korotkov. During his leadership in the late 90s of the XX century, the basic principles of Bioelectrography were developed. A technique for shooting gasdischarge images of human fingers was developed. In addition, a software and hardware complex GDV-camera was created that performs such shooting and computer processing of GDV-grams. The possibility of creating computer programs for analyzing the psychoemotional and physiological state of a person was based on a diagnostic method developed at the end of the XX century by the German doctor Peter Mandel. Based on a large body of data from clinical studies, Peter Mandel found that certain changes in the corona of light around the fingers correspond to the state of certain organs and systems of the body. Changes in the corona of light in its individual sectors indicated changes in the functional state of a person, his psychoemotional characteristics at the time of the study.

The development of Bioelectrography in the XXI century is associated with the expansion of the use of this method in various fields: medicine and cosmetology, sports, law enforcement, agriculture, psychology and pedagogy $[9,10,11,12]$. The last two areas are particularly relevant for our research.

The psychological aspect of using the gas-discharge visualization method is widely presented in the studies of authors from different countries of the world. A number of programs-GDV-diagram, GDV-chakra, developed under the guidance of K. G. Korotkov, were directly aimed at identifying and evaluating the features of a person's psychoemotional state, his stress resistance, and features of emotional response in various life situations. They were used for studying the features of the personal emotional state and even groups of people (together with a special GDV Sputnik antenna) during lectures, theater performances and concerts, as well as meditations.

In the studies of S. G. Jura and M. I. Yanovsky was the psychological aspects of using the method of gas discharge visualization for the understanding of the impact of meditation on the human body. In addition, there was the reactions to the presentation of color and olfactory impulses, systematic scope of application of GDV graphics in various fields of human activities [12].

The study of T. E. Bulatova largely contributed to the inclusion of the gas-discharge visualization method in pedagogical research. Using traditional psychological research methods along with the method of GDV diagnostics, T. E. Bulatova showed a high correlation of data on the psychophysiological and energy state of schoolchildren obtained using traditional psychological methods and GDV graphics. The results of the study T.E. Bulatova confirmed the effectiveness of including the gas-discharge visualization method in psychological and pedagogical research to obtain reliable information about the psychophysiological state of schoolchildren. Its changes depends on the teaching methods and organization of the educational process. She noted the availability and simplicity of diagnostics using the GDV camera, the ability to assess the features of not only the physical but also the mental state of schoolchildren, the effectiveness of innovations and healthsaving technologies used.

It should be noted that there are very few studies related to using of the gas-discharge imaging in educational research. This is a new direction in using of Bioelectrography. We could not find any special works that reveal the possibility of using the gas-discharge visualization method in the field of speech therapy, with the exception of one work by M. L. Baranova, which considers the possibility of including instrumental methods in the work of a speech therapist, including a GDV camera.

\section{Methods}


Our research was aimed at studying the possibility of using the GDV-graphy method for efficiency assessment of the correction, which aimed at overcoming statokinetic functions violations in younger schoolchildren with phonetic and phonemic speech disorders. Statokinetic functions violation is very often detected in children with phonetic and phonemic speech disorders. This fact is indicated in the numerous studies.

The experiment involved 20 students in grades I-II of primary school in Rostov-on-Don.

The study had two directions. The first direction of diagnostics was associated with the identification of features of the statokinetic functions development in primary school children with speech disorders. The second direction of diagnostics was aimed at identifying the features of the functional state of the organism and the functional training influence using correctional stabilographic methods on the schoolchildren's psychophysiological state [13].

In the first direction, the software and hardware complex "Stabilan-01", based on the method of stabilography, was used to assess statokinetic functions. We used diagnostic stabilographic techniques such as the Romberg test and the "Target" test. During the diagnostic tasks, the sensitive platform on which the person was located, detected and analyzed all changes in the position of his body in space. The person had the diagnostic tests with opened and closed eyes with optical and acoustic stimulation and adjusted the body position in space by holding the marker in the center of the target. Parameters such as static and dynamic balance, and hand-eye coordination were analyzed. The analysis of the obtained results was presented in the form of stabilograms that clearly reflect the existing violations of the student's statokinetic functions.

Actually, the state of balance is provided by constant movement of the body and depends on the coordinated work of the visual and vestibular organs, the muscular system and the regulating activity of the central nervous system. Therefore, the state of the equilibrium function is directly related to the psychophysiological state of the body. This fact was important for our study. Thus, the software and hardware complex "Stabilan-01" allowed us to assess in detail the characteristic features of the state of statokinetic functions in children with speech disorders.

In the second direction of diagnostics, we used the method of GDV-graphy for the general assessment of the energy state and functional capabilities of the schoolchildren body with speech disorders, as well as the impact of functional training using the software and hardware complex "Stabilan-01" on the schoolchildren's psychophysiological state. Durind receiving GDV-grams of glow, it is also possible to identify children with vegetative instability and reduced adaptation. We used several GDV programs:

- GDV Capture program, which provided the process of shooting GDV-grams with ten fingers of the subjects;

- GDV Energy Field program, which allows you to assess the features of the energy state of the subjects;

- The GDV Diagram program is designed to assess the functional state of the body, the level of adaptation and stress resistance, as well as the psychoemotional state of the person (analysis parameters - activation coefficient and integral entropy).

We took pictures with ten fingers of each person's hands.

The photography of GDV-grams were carried out in two modes: with filter and without filter. In the unfiltered mode, the programs recorded the current psycho-physiological and psycho-emotional state of the subjects. In a filtered mode, a filter made of a special plastic film was used, which was applied to the lens of the lens and compensated for changes caused by the activation of the autonomic nervous system. At the same time, the characteristics of the body's functional state that are not related to current external or internal influences were recorded. 


\section{Discussion of results}

Analysis of the results of statokinetic functions diagnostics of primary school children with speech sound disorders allowed us to come to the following conclusions. $20 \%$ of children in the experimental group had a low level of statokinetic functions. Taking the diagnostic tests by children with low indicators was characterized by instability of posture. In addition, taking motor tasks were performed with deviations in the anterior-posterior and lateral directions, movements were incomplete in volume. It is one of the reason, why in some cases children were absent-minded. In addition, they had misunderstanding of instructions.

$40 \%$ of the examined children were characterized by an average level of statokinetic functions development.

This group of children was also the lack of stability of posture when carrying out diagnostic tests. Deviations in the antero-posterior and lateral directions were less pronounced than in children with low statokinetic functions.

Another $40 \%$ of the children who participated in the experiment had a high level of statokinetic function development. They were successful with the tasks of concentration to maintain a static posture, coordination of movements in space and posture regulation (Table $1)$.

Table 1. The level of development of statokinetic functions at the initial stage of the study (in percent).

\begin{tabular}{|c|l|l|l|}
\hline Level & High level & $\begin{array}{l}\text { Average } \\
\text { level }\end{array}$ & Low level \\
\hline Number of children & 40 & 40 & 20 \\
\hline
\end{tabular}

It should be noted that the lowest indicators in the development of statokinetic functions were found in children with severe speech disorders. A certain dependence of the level of development of motor and speech functions was revealed.

The results of the energy state assessment and functional capabilities of the body in schoolchildren with speech disorders using the GDV camera revealed the following statements. According to the parameter "activation coefficient", the pupils were divided into 5 groups, each of which accounted for $20 \%$ of the total number of pupils. The first group of children was found to be in a normal emotional state (activation coefficient 2.04.0). The second group was characterized by a concentrated active state (activation coefficient 4.0-4.5). These two groups of children showed a high level taking stabilographic tests. The third group of children was characterized by a low activation coefficient $(0.5$ 1.5), deconcentration of attention and inhibited state. During the tests, an incomplete range of movement, reduced concentration characterized these children and, as a result, difficulties in maintaining posture. Two more groups of children showed a high and very high level of activation coefficient. Children with a high activation coefficient (6.0-8.0) were characterized by a nervous state, increased anxiety, which they could not cope with. Moreover, in children with very high activation coefficients (8.0-10.0), the excited state reached an emotional peak, approaching a stressful state. These children were characterized by the greatest deviations in the anterior-posterior and lateral directions during stabilographic tasks, inattention and misunderstanding of instructions.

According to the integral entropy index, $20 \%$ of the pupils showed normal resistance to loads (integral entropy 1.5-1.9). High indicators in the diagnosis of statokinetic functions also characterized this group of children. $60 \%$ of children had hypersensitivity to loads (integral entropy 1.1-1.5), and another $20 \%$ of the examined children had hypersensitivity to loads (integral entropy up to 1.1). According to the integral entropy index, the study of 
statokinetic functions showed mainly medium and low indicators in children of the second and third groups.

Thus, our results indicate that the level of statokinetic functions development in most cases corresponds to the same level of energy and functional state of children with speech disorders.

Based on the results of diagnostics, we developed individual correction programs for schoolchildren participating in the experiment, including functional training of statokinetic functions using the software and hardware complex "Stabilan-01". Functional training included tasks such as "Building pictures", "Balls", "Cubes". Students had to control the movement of their body, collected split images, sent the ball to the pocket of the corresponding color, and collected cubes. The number of functional training sessions for the statokinetic functions development in each student was determined individually, in accordance with the results of the previous diagnosis. The level of difficulty of completing tasks was also determined individually.

The effectiveness of functional training was monitored after each lesson for possible adjustments to the level of complexity of the task, as well as at the end of the entire course. After completing the course of functional training, $60 \%$ of children showed a high level of development of statokinetic functions. Significantly increases the concentration and the ability to self-regulate during stabilographic tasks. In $40 \%$ of children, statokinetic functions were at an average level. At the final stage of the experiment, we did not identify children with a low level during the stabilographic test (Table 2).

Table 2. The level of development of statokinetic functions at the final stage of the study (in percent).

\begin{tabular}{|l|l|l|l|}
\hline Level & High level & $\begin{array}{l}\text { Average } \\
\text { level }\end{array}$ & Low level \\
\hline $\begin{array}{c}\text { Number of } \\
\text { children }\end{array}$ & 60 & 40 & - \\
\hline
\end{tabular}

The results of the assessment of the energy and functional state of the pupils also indicated existing changes. Therefore, according to the indicator "activation coefficient", there were no children with a very high level of activation at the final stage of assessment (8.0-10.0). There were also no children with a low activation coefficient (0.5-1.5). Most of them were children with increased activity $-60 \%$ of the total number of pupils. Their excitement and some tension were related to the desire to complete the task as best, as possible, and not to fear or uncertainty. Another $40 \%$ of children had a concentrated active state in terms of activation coefficient (4.0-4.5). Data on integral entropy showed an increase in the group of children with normal load sensitivity, there was $60 \%$ of pupils (integral entropy $1.5-1.9$ ). Another $40 \%$ of children were found to be hypersensitive to stress (integral entropy 1.1-1.5). There were no children with increased sensitivity to stress among the subjects.

\section{Conclusions}

The current stage of development of psychological and pedagogical research is characterized by the active inclusion in the diagnostic process of software and hardware complexes. They ensure the efficiency, reliability and visibility of obtaining the necessary data on the studied parameters. One of these modern software and hardware complexes is a GDV-camera that works based on the gas-discharge visualization method (Kirlian effect). The use of GDV-graphy makes it possible to assess the energy and functional state of 
children, which helps to individualize the correctional program, taking into account the psychophysiological characteristics of the child and evaluate its effectiveness. The data obtained in the analysis of GDV-grams correspond to the indicators obtained in the diagnosis and monitoring of the level of statokinetic functions development. This fact allows us to assess positively the possibility of including the method of GDV-graphy in psychological and pedagogical research.

\section{References}

1. A. Akhmetzyanova, WASJ, 29(6), 743-746 (2014)

2. A. Chinkin, VZNU. BS, 2, 189-201 (2014)

3. E. Bushinskaya, MER, 3, 15-18 (2014)

4. I. Vlasova, The Youth of the 21st Century: Education, Science, Innovations. Proceedings of VI International Conference for Students, Postgraduates and Young Scientists, 395-398 (Vitebsk state University, 2019)

5. A. K. Ho, K. Wilmut, HMS, 29(4), 605-614 (2010)

6. F. Tahavieva, I. Nigmatullina, Astra Salvensis, 5(10), 315-322 (2017)

7. K. Korotkov, M. Shustov, Kirlian Effect-past and present (Saint Petersburg-Tomsk, 2017)

8. K. Korotkov, Bioelectromagnetic and Subtle Energy Medicine, 111-121 (CRC Press, 2015)

9. I. Korobka, E. Yakovleva, T. Zarubina, K. Korotkov, SAMBS 11( 3), 572-579 (2012)

10. Bioelectromagnetic and Subtle Energy Medicine (CRC Press, 2015)

11. I. Ciesielska, TRJ, 5(80), 216-225 (2010)

12. S. Jura, Ethical algorithms of the universe (LAMBERT Academic Publishing, 2014)

13. A. Kobrin, N. Babanov, 2018 4th International Conference on Information Technologies in Engineering Education, Inforino 2018, 4, 8581770 (2018). 